\title{
La noción de "justificación”, ¿un concepto dual?
}

\author{
ÁNGELES ERAÑA \\ Instituto de Investigaciones Filosóficas \\ Universidad Nacional Autónoma de México \\ angeleserana@yahoo.com
}

\begin{abstract}
Resumen: Dos posiciones dominantes en el ámbito de las teorías sobre la justificación epistémica son el "internismo" y el "externismo". Se afirma que las intuiciones subyacentes a cada una de ellas son contradictorias y, por tanto, no es posible hacerlas converger en una sola teoría. Haré ver que esto último no sólo es posible, sino necesario. Para ello apelaré a la teoría dual de sistemas y argumentaré que si (1) como afirma esta teoría, las habilidades de razonamiento de los seres humanos están estructuradas en dos sistemas diferentes, entonces (2) tenemos buenas razones para afirmar que el estatus que el concepto de "justificación" les provee a las creencias es dual, i.e., ofrece criterios de evaluación que deben utilizarse de manera diferenciada para creencias de distintos tipos, las cuales son resultado de cada uno de nuestros sistemas de razonamiento.
\end{abstract}

Palabras clave: internismo/externismo, razonamiento, racionalidad, normatividad epistémica

\begin{abstract}
Epistemic "internalism" and "externalism" have been two dominant positions within justification theories. Intuitions underlying these positions are held to be contradictory and, thereby, it is not possible to make them converge into a unified theory. I will show that this is not only possible, but necessary. In order to do this, I will appeal to the Dual System Theory, and I will argue that if, (1) as this theory asserts, our reasoning abilities are structured into two different systems, then (2) we have good reasons to assert that the normative status that the notion of "justification" provides to our beliefs is dual, i.e., it offers evaluation criteria that should be differentially used for different kinds of beliefs, which are the result of each of our reasoning systems.
\end{abstract}

Key words: internalism/externalism, reasoning, rationality, epistemic normativity

\section{Introducción}

Una pregunta central de la epistemología es ¿cuáles son los factores que determinan cuándo una creencia está epistémicamente justificada? En la literatura encontramos diferentes respuestas para ella, muchas de las cuales han sido agrupadas bajo los rubros de "internismo" (i.e., sólo las creencias u otros estados internos al sujeto sirven para determinar cuándo está justificada una creencia) en oposición al "externismo" 
(i.e., la justificación de una creencia está determinada por su relación con el estado externo de cosas).

En este trabajo argumentaré que el internismo y el externismo epistémicos rescatan (y describen) hechos normativos distintos, cada uno de los cuales está asociado con criterios de evaluación diferentes que deben utilizarse de manera diferenciada para creencias de distintos tipos. Para sostener este argumento utilizaré la interpretación que hace la llamada teoría dual de sistemas (TDS) de la evidencia empírica que arroja una serie de estudios llevados a cabo en las últimas décadas sobre nuestros procesos de razonamiento.

La TDS es una teoría (descriptiva) sobre la estructura del razonamiento humano y el tipo de mecanismo que subyace a él. En particular, postula la existencia de dos sistemas de razonamiento, cada uno de los cuales produce diferentes tipos de resultado frente a las mismas tareas de razonamiento. Haré ver que la operación de cada uno de estos sistemas está asociado con los hechos normativos descritos, respectivamente, por el internismo y el externismo de la justificación epistémica. $\mathrm{Si}$ esto es correcto, entonces tenemos buenas razones para afirmar que la noción de justificación es un concepto dual que puede ser entendido apropiadamente si hacemos un análisis más fino de nuestras habilidades reales de razonamiento.

\section{El internismo epistémico en oposición al externismo epistémico}

Antes de presentar mi argumento es importante esbozar algunas de las tesis centrales del internismo y del externismo epistémicos. Esto me permitirá hacer ver que la diferencia sustancial entre tales tesis radica en que el estatus normativo que, según cada una de ellas, le confiere el concepto de "justificación" a nuestras creencias es de distinto tipo.

\subsection{El internismo de la justificación}

La tesis del internismo epistémico puede formularse como sigue: aquello que determina si una creencia está justificada son factores o estados internos del sujeto de la creencia. Es común que los internistas recurran a las siguientes dos premisas para sostener esta idea: (1) una creencia está justificada sólo si tenemos buenas razones para aceptarla; (2) sólo los estados internos del sujeto (e.g., otras creencias u otros estados mentales) pueden ser considerados razones para creer (o para aceptar una creencia).

La formulación de esta tesis y de las premisas que la sustentan es lo suficientemente general como para admitir distintas interpretaciones. 
Aquí sólo me centraré en una versión del internismo según la cual un estado o factor es interno de un sujeto $(S)$ si $S$ puede acceder a él a través de la reflexión (i.e., un internismo accesibilista). La idea de fondo es que los factores que determinan la justificación de una creencia son condiciones a las cuales el sujeto $(S)$ tiene (o puede tener) acceso cognitivo directo, i.e., si $S$ se pregunta si una condición $(C)$ se da, entonces $S$ cree que $C$ se da si y sólo si $C$ se da. El internista afirma que es dudoso que los seres humanos podamos tener acceso de este modo a hechos o estados del mundo y, por tanto, debemos aceptar que sólo nuestras creencias (y otros estados mentales) pueden ser considerados como razones para creer y, en este sentido, sólo ellas pueden proveer justificación a otras creencias.

Un internismo accesibilista como éste parte de la aceptación de una noción de la justificación como guía, i.e., las condiciones para la justificación deben guiar a la gente en sus decisiones respecto de qué creer (BonJour 2005, p. 236), y está sustentado en un concepto deontólogico de la justificación, i.e., la creencia $p$ de $S$ está justificada si, al aceptarla, $S$ cumple con su deber (u obligación) epistémico. Desde esta perspectiva, un sujeto $S$ está (epistémicamente) obligado a creer que $p$ sii: (i) $p$ es probablemente verdadera a la luz de la evidencia total de $S$; (ii) el hecho de que $S$ crea que $p$ no es incompatible con que $S$ crea una proposición $q$ que es probablemente verdadera dada la evidencia total de $S$ y que es epistémicamente más importante que $p$; y (iii) $S$ es capaz de reconocer la verdad de (i) a través de la reflexión sobre su evidencia (Moser 1956, p. 218). Estas condiciones implican que una creencia sólo estará justificada si el sujeto de la creencia evalúa su aceptabilidad dadas sus otras creencias y su evidencia y si, una vez hecho esto, puede reconocer la (probable) verdad de la creencia y puede, por tanto, defender la (probable) verdad de su creencia frente a sí mismo y a otros interlocutores. Si esto último es correcto, entonces no es suficiente que $S$ tenga ciertas razones para creer que $p$; su creencia de que $p$ sólo estará justificada si $S$ puede ofrecer razones a favor de ella.

Nótese que esta noción de "justificación" no sólo requiere que la creencia justificada tenga ciertas propiedades justificativas, sino también que el sujeto de la creencia pueda tener acceso a esas propiedades y pueda dar cuenta de ellas. En otras palabras, los requisitos asociados al concepto deontológico de justificación imponen exigencias al sujeto de la creencia, i.e., éste debe ser capaz de defender su creencia. En este sentido, dice Kornblith (2001): 
lo que se requiere para que una persona esté justificada en sostener una creencia es que esa persona tenga cierta justificación para la creencia, donde tener una justificación se identifica típicamente con estar en una posición, en algún sentido pertinente, para producir un argumento para la creencia en cuestión. (p. 2)

Este planteamiento es consistente con la noción de justificación como guía, según la cual los criterios de justificación deben guiar al sujeto para saber cuándo debe aceptar una creencia particular. El supuesto subyacente a esta idea es que las relaciones entre nuestras creencias (entre ellas y otros estados mentales, y entre ellas y el mundo) están gobernadas por un conjunto de principios normativos que determinan cuándo está epistémicamente permitido aceptar una nueva creencia. Estos principios constituyen un ideal regulativo al que debe apegarse un sujeto si quiere tomar una decisión correcta con respecto a qué creer.

Si lo dicho hasta aquí es correcto, entonces la tesis particular del internismo accesibilista puede formularse como sigue: la creencia de que $p$ de un sujeto $S$ está justificada sólo si $S$ puede (al menos potencialmente) ofrecer un argumento a favor de $p$ (esto garantiza -en un grado apropiado- la verdad de $p$ y supone que $S$ ha seguido las normas asociadas a los principios normativos de razonamiento).

\subsection{El externismo de la justificación}

Una de las versiones más aceptadas y generalizadas del externismo (i.e., el confiabilismo epistémico) parte de una concepción consecuencialista de la justificación, i.e., los seres humanos tenemos fines epistémicos $\mathrm{y}$ estamos justificados en creer que $p$ si la aceptación de $p$ es conducente a la obtención de esos fines (Plantinga 1993, p. 27). Aquí me ocuparé de una posición aún más específica (i.e., el confiabilismo verídico), según la cual el fin epistémico por excelencia es la verdad.

Desde esta perspectiva, la justificación es esencialmente una cuestión de que mis creencias hayan sido producidas o se sostengan por un proceso cognitivo confiable (i.e., que produzca un mayor número de creencias verdaderas que falsas).

Un proceso de producción de creencias es, según Goldman (1979):

una operación o procedimiento funcional, i.e., algo que genera un mapeo de ciertos estados — los "insumos" - a otros estados — los "resultados"Los estados en el caso presente son estados de creer ésta o aquella proposición en un momento dado. (p. 299) 
Podría pensarse que esta manera de analizar la noción de "proceso de formación de creencias" nos lleva de vuelta hacia una visión internista de la justificación: los determinantes de la justificación son (en última instancia) estados internos de un sujeto (e.g., procedimientos que tienen lugar "en la cabeza" de los individuos; otras creencias que sirven como insumo, creencias y otros estados mentales que se relacionan inferencialmente entre sí para producir una nueva creencia, etc.). La única diferencia con el internismo parecería ser que el juicio de aceptabilidad epistémica no está sustentado en cada instancia de creencia producida, sino en la de un tipo de operaciones que tienden a producir un mayor número de instancias de creencias verdaderas que falsas. Sin embargo, ésta sería una apreciación incorrecta de lo que establece el confiabilismo.

Como Goldman afirma (1979, p. 297), cualquier principio de justificación correcto debe ser un principio que haga requerimientos causales, i.e., el tipo de relación causal que haya entre la creencia y su objeto debe desempeñar un papel en determinar cuándo una creencia está justificada. Así, el confiabilismo se entiende mejor si éste se formula del siguiente modo: lo que determina la justificación de una creencia es la confiabilidad del proceso $(P)$ que la produce, donde esta confiabilidad depende de un hecho estadístico, i.e., sólo si $P$ produce una mayor proporción (o un mayor porcentaje) de creencias verdaderas que falsas, $P$ puede ser considerado confiable. Si esto es correcto y si aceptamos que aquello que determina la verdad de una creencia (o la tendencia de un proceso para producir creencias verdaderas) es su relación con el estado de cosas en el mundo, entonces podemos aceptar que los factores determinantes de la justificación no tienen que ver con las creencias del sujeto respecto de la confiabilidad del proceso, ni con la relación de su creencia actual con el resto de sus creencias. Más bien, ellos provienen de que haya una relación adecuada de la creencia con aquello que la causó o de que el proceso de producción de creencias tienda a establecer este tipo de relaciones entre las creencias que produce y los hechos del mundo. Esto último garantizará (en cierto grado) la confiabilidad del proceso y, así, la obtención de creencias verdaderas (Kornblith 2001, p. 2).

Si aceptamos estas ideas, podemos afirmar que (al menos algunas de) las propiedades justificativas de una creencia son producidas por cierto tipo de relación causal que involucre de forma apropiada al sujeto y al objeto de la creencia. Estas propiedades no necesariamente son (y posiblemente nunca lo sean) accesibles al sujeto de la creencia. Así, el externismo aleja el foco del problema de la justificación de la pregunta 
por nuestra capacidad para ofrecer argumentos que nos permitan defender intelectualmente nuestras creencias y lo acerca a la cuestión de estar en una relación causal apropiada con el medio ambiente.

La posición del confiabilismo verídico puede formularse como sigue: una creencia $p$ puede considerarse como una creencia justificada sólo si $p$ fue producida por un proceso confiable, donde el insumo sobre el que éste opera determina, en cierto grado, el resultado específico que entregará. ${ }^{1} \mathrm{El}$ insumo, en este sentido, causa la creencia $p$ del sujeto $S, \mathrm{y}$ sólo si $p$ está en una relación causal apropiada con el ambiente externo, el proceso operará correctamente, i.e., producirá creencias verdaderas como resultado.

\section{3. La oposición internismo-externismo y los estatus normativos}

El internismo y el externismo han sido considerados por algunos autores como dos cuernos de un dilema: o bien las fuentes de la justificación son estados o condiciones cognitivamente accesibles a la persona cuyas creencias están siendo justificadas, o bien no lo son. Sin embargo, otros autores han argumentado que éstas no constituyen un auténtico dilema puesto que se dirigen a preguntas distintas.

Richard Foley (2002), por ejemplo, afirma que el internismo y el externismo de la justificación no son posiciones en competencia porque se ocupan de problemas distintos: mientras que el segundo está preocupado por responder a la pregunta de ¿qué es el conocimiento?, el primero está interesado en responder a la pregunta de ¿cuándo un sujeto tiene razones para creer que $p$ ? Así, afirma este autor, estas dos posiciones trazan proyectos distintos: el internismo busca una explicación de lo que se requiere para "poner en orden nuestra casa intelectual" y el externista busca una explicación de lo que se requiere para estar en una relación de conocimiento con el medio ambiente (Foley 2002, p. 180).

Aun si aceptásemos que las preguntas que se desprenden de estas dos posiciones son distintas, hay otra más fundamental que subyace a las dos, i.e., ¿cuál es el estatus normativo que la "justificación" confiere a nuestras creencias? Un estatus normativo es una propiedad (o un conjunto de propiedades) que puede atribuírsele a las creencias sólo si éstas satisfacen ciertos estándares o se ajustan a ciertas normas que

${ }^{1}$ Es importante mencionar que, según Goldman, los procesos de producción de creencias no sólo reciben otras creencias (u otros estados mentales del sujeto) como insumo, sino también (y de manera muy común y muy importante) hechos del mundo. 
establecen la diferencia entre una creencia epistémicamente aceptable y otra que no lo es (Williams 2001).

Si el debate se formula en estos términos, entonces es razonable afirmar que el internismo y el externismo comparten preocupaciones; pero el dilema parecería presentarse de nuevo. Acabo de decir que un estatus normativo es una propiedad (o un conjunto de propiedades) que sólo puede ser atribuida a una entidad $(E)$ si $E$ satisface la norma (o los estándares) que determina(n) las condiciones de satisfacción del estatus (o las condiciones de aplicación de la propiedad). Sin embargo, en el caso de la "justificación", las normas que se desprenden del internismo y del externismo establecen cosas distintas:

Norma internista: Si $S$ está justificado (en $t$ ) en creer que $p$, entonces $S$ (en $t$ ) debe creer (o creer justificadamente o saber) que $p$ está justificada.

Norma externista: Si $S$ está justificado (en $t$ ) en creer que $p$, entonces la creencia $p$ de $S$ debe haber sido formada por un proceso confiable.

Nótese que mientras la primera norma sólo puede cumplirse si $S$ tiene creencias sobre la justificación de su creencia de que $p$ (i.e., si $S$ tiene creencias sobre las condiciones que deben satisfacerse para estar justificado en creer que $p$ y, por tanto, si puede acceder a dichas condiciones), la segunda no impone este requisito (no necesitamos tener ninguna creencia sobre la confiabilidad de nuestros procesos para que las creencias producidas por ellos estén justificadas).

En otras palabras, la primera norma al mismo tiempo (1) determina las condiciones que debe satisfacer una creencia para estar justificada y (2) establece ciertas obligaciones para el sujeto (e.g., el sujeto debe creer (o creer justificadamente, o saber) que su creencia satisface las condiciones mencionadas). La segunda norma, por su parte, no dice nada respecto a (2): según esta norma, no es necesario que el sujeto de la creencia $S$ sepa (o crea, o crea justificadamente) que es confiable el proceso de producción de creencias con el que llegó a creer que $p$ para que su creencia de que $p$ esté justificada y, por lo tanto, los estándares de justificación no obligan al sujeto a tener creencias con respecto a (la confiabilidad de) su proceso de formación de creencias para que la creencia esté justificada.

Este contraste pone en claro el hecho de que las diferencias entre estas posiciones apuntan no solamente a las fuentes de la justificación (creencias frente a relaciones causales o procesos de producción 
de creencias), sino también al tipo de propiedad que se atribuye a una creencia cuando se afirma que ella "está justificada": en el caso del internismo, la propiedad de "estar justificado" es relacional y proviene de una relación entre estados internos del sujeto; en el caso del externismo, la propiedad es estadística y depende de una relación causal apropiada entre creencias y estados del mundo (i.e., estados externos a los cuales el sujeto puede no tener acceso).

La pregunta que surge es si sólo uno de estos estatus (o propiedades) captura adecuadamente la noción de "justificación". En lo que sigue argumentaré que esto no es el caso y que, por lo tanto, aun si el debate se formula en estos términos, no tiene por qué presentarse el dilema de la justificación. Para sustentar mi argumento recurriré a la evidencia empírica que arrojan algunos estudios recientes en psicología cognitiva del razonamiento. ${ }^{2}$

\section{La TDS y los estatus normativos}

Desde los años sesenta, la psicología cognitiva del razonamiento ha estado preocupada por entender el vínculo que existe entre el tipo de procedimiento que utilizamos los seres humanos en nuestro razonamiento cotidiano y las exigencias impuestas por las teorías normativas de la racionalidad. Por esta razón, los psicólogos del razonamiento han diseñado y llevado a cabo una serie de estudios que pretenden diagnosticar qué procesos cognitivos subyacen a nuestro razonamiento y cómo responden ellos a las exigencias clásicas de racionalidad (i.e., un sujeto es racional si (y sólo si) razona de acuerdo con los principios normativos de razonamiento correcto, e.g., los principios de las

${ }^{2}$ Como bien me ha señalado un dictaminador anónimo, el debate que aquí presento podría formularse en términos de la oposición entre naturalismo y normativismo. Es importante señalar que uno de los supuestos que subyace a mi presentación es que la epistemología es una disciplina esencialmente normativa, i.e., los conceptos propiamente epistémicos (como el de "justificación") no son sólo estados o condiciones factuales, sino estatus normativos particulares (Williams 2001). Así, una de las preocupaciones centrales del epistemólogo naturalista tendría que ser dar respuesta a la pregunta acerca de ¿cuál es el estatus que la "justificación" provee a nuestras creencias? Como he argumentado en otro lugar (Eraña 2007), la respuesta que algunos naturalistas ofrecen a esta pregunta entra en conflicto con la que el normativismo ofrecería. Así, es razonable afirmar que son distintas las propiedades que cada una de estas posiciones asocia con estar "justificado" en creer que $p$, y, por tanto, la pregunta acerca de si sólo un conjunto de ellas captura adecuadamente el hecho de que las creencias estén justificadas seguiría vigente bajo esta formulación del debate.

Diánoia, vol. LIV, no. 62 (mayo 2009). 
lógicas formales o del cálculo de probabilidades). ${ }^{3}$ El comportamiento raciocinativo observado en estos estudios tiende (sistemáticamente) a la violación de los principios normativos mencionados. Este hecho ha recibido diferentes interpretaciones y ha generado un gran debate con respecto a qué significa "ser racional" (Stein 1996; Cohen 1986; Lopes 1991). En lo que sigue sólo examinaré una interpretación muy reciente de estos resultados, i.e. aquella que se desprende de la TDS.

\subsection{La teoría dual de sistemas}

Como recién mencioné, un número importante de psicólogos cognitivos del razonamiento han llevado a cabo una serie de estudios para diagnosticar el tipo de proceso que subyace a nuestro razonamiento cotidiano. En la mayoría de estos estudios se pide a los sujetos encuestados que evalúen la probabilidad de que cierto suceso ocurra (dada cierta información previa) o la validez de cierta regla o argumento y, en la mayoría de ellos, los sujetos hacen una evaluación incorrecta. ${ }^{4}$ Las únicas dos conclusiones que han generado cierto consenso entre los psicólogos son: (1) los seres humanos sistemáticamente cometemos errores al razonar y (2) estos errores se deben, al menos en parte, a que nuestros procesos de razonamiento involucran sesgos (i.e., fuentes de desviación sistemática de los principios normativos del razonamiento). ${ }^{5}$

La TDS surge como un intento para explicar estas conclusiones. Según esta teoría, la capacidad humana de razonamiento opera a través de diferentes tipos de procesos que se agrupan en y constituyen dos

${ }^{3}$ Tversky y Kahneman 1983; Wason 1966; Wason y Johnson-Laird 1970; Evans 1984; Kahneman et al. 1982.

${ }^{4}$ Por ejemplo, a un grupo de sujetos se les pide que estimen la probabilidad de que Juan sea ingeniero con base en la siguiente información: Juan es miembro de un grupo de cien personas elegidas al azar, setenta de las cuales son ingenieros y treinta abogados. Además, se provee la siguiente descripción de Juan: "es un hombre de treinta años. Está casado y no tiene hijos. Tiene muchas habilidades y motivaciones, de manera que promete ser exitoso en su campo. Tiene buenas relaciones con sus colegas" (Tversky y Kahneman 1982). Dada la información, la expectativa es que los sujetos estimen la probabilidad de que ocurra un suceso con base en las probabilidades iniciales, i.e., 0.7 es la probabilidad de que Juan sea ingeniero. Sin embargo, la mayoría de los sujetos respondió que era de $0.5 \mathrm{y}$, en general, el juicio emitido apelaba a la semejanza de la descripción de Juan con algún estereotipo de los ingenieros (o de los abogados).

${ }^{5}$ La idea de fondo en este planteamiento es que las desviaciones (o los errores) provocadas por los sesgos no son errores transitorios de procesamiento, sino que se deben a la constitución misma de nuestros procesos de razonamiento (Stanovich y West 2000). 
sistemas cognitivos separados (Evans 2006). Ambos sistemas - 1 y S2- son inferenciales (i.e., nos permiten producir resultados informacionales con base en recursos informacionales previos), pero cada uno de ellos tiene una historia evolutiva propia (Evans 2003) que les ha provisto con propiedades diferentes. Veamos.

La sistematicidad (i.e., aun después de haber recibido una explicación de por qué la respuesta emitida a un problema de razonamiento constituye un error, los sujetos vuelven a emitir respuestas del mismo tipo) y la universalidad (i.e., el mismo tipo de error es cometido por sujetos pertenecientes a distintas culturas o comunidades) de los errores cometidos por los sujetos en las tareas de razonamiento nos dan buenas razones para pensar que hay una forma universal de cognición (i.e., compartida por los seres humanos). Ésta, según la TDS, descansa en procedimientos de optimización que permiten "que ciertos actos muy complejos de procesamiento de información tengan lugar muy rápidamente" (Evans y Over 1996, p. 135) aunque no siempre entreguen resultados acordes con los principios normativos de razonamiento. Estos procedimientos o procesos constituyen un sistema -el S1-que tiene las siguientes características (Evans y Over 1996; Sloman 1996; Stanovich y West 2000): es computacionalmente poderoso; rápido; automático (i.e., sus respuestas persisten aun si el individuo trata de ignorarlas); innato, pero modelado por nuestra interacción con el medio ambiente; no exige mucha capacidad cognitiva; lo guían consideraciones de pertinencia dirigidas a inferencias intencionales a través del uso de implicaturas conversacionales, incluso en situaciones que no tienen características conversacionales (Evans 2003), i.e., tiende a contextualizar y personalizar la información que recibe y, por tanto, es muy útil para la socialización.

Por otro lado, una serie de estudios más detallados (Stanovich y West 2000) hicieron ver que sujetos con mayores niveles de educación se equivocan menos frecuentemente que quienes tienen niveles más bajos de educación. Esto ha servido a la TDS para sostener que el aprendizaje hace posible una forma más sofisticada de cognición que utiliza la información almacenada en la memoria para llevar a cabo inferencias y que utiliza el pensamiento abstracto e hipotético. Los procesos subyacentes a esta forma de cognición constituyen, según la TDS, otro sistema -el S2- que probablemente tiene una historia evolutiva más reciente propia de los seres humanos. Este sistema se caracteriza por ser voluntario (i.e., controlado); por responder a instrucciones verbales; por ser analítico, lento; requiere hacer un gran esfuerzo cognitivo y dejar de atender otras cuestiones; y está basado en reglas. Además, 
este sistema tiene una baja capacidad de procesamiento (en parte porque demanda mucha capacidad cognitiva) y tiende a descontextualizar y despersonalizar la información que recibe.

Según la TDS, estos dos sistemas compiten constantemente por la información disponible y, dadas sus características, es altamente probable que el primero emita respuestas inmediatas y que sea más apto para procesar cargas computacionales pesadas. Pero el hecho de que sea guiado por consideraciones de pertinencia lo lleva a ofrecer (sistemáticamente) respuestas equivocadas desde una perspectiva normativa. El otro sistema - S2 - es más lento, pero es capaz de descontextualizar la información para procesarla en términos de reglas y principios. Además, aunque $\mathrm{S} 1$ es computacionalmente más poderoso, S2 puede corregir e inhibir las respuestas que entrega S1 por default (Evans y Over 1996; Sloman 1996), con lo cual le permite al sujeto satisfacer los requisitos clásicos de racionalidad.

Estas ideas llevaron a Evans y Over (1996) a afirmar que si queremos entender cómo nuestros procesos se comportan frente a los requisitos de racionalidad, entonces tenemos que renunciar a la idea de que la "racionalidad" puede analizarse desde un único punto de vista. Según estos autores, para este fin es necesario introducir una diferencia terminológica entre dos maneras de entender lo que significa "ser racional": hay una noción de racionalidad ( racionalidad $_{1}$ ) que sirve para evaluar los fines de un sujeto y su aptitud para alcanzarlos; y otra (raciona$\operatorname{lidad}_{2}$ ) que evalúa la satisfacción de ciertos estándares normativos de racionalidad. Veamos cómo estas ideas nos llevan de vuelta al debate entre el internismo y el externismo de la justificación.

\subsection{La racionalidad y la justificación epistémica}

Para mostrar el vínculo que hay entre la TDS y el debate de la justificación epistémica es necesario aceptar los siguientes dos supuestos (prevalecientes en la literatura epistemológica): (a) una explicación de la justificación epistémica ofrece, por sí misma, una explicación de la creencia racional (i.e., los requisitos que debe satisfacer una creencia para estar justificada determinan, al mismo tiempo, la racionalidad de la creencia) y; (b) un sujeto es racional si la mayor parte de sus creencias son racionales. Si (a) y (b) son aceptadas, entonces es razonable afirmar que los criterios que debe satisfacer una creencia para considerarla justificada sirven también para determinar cuándo un sujeto es racional (i.e., un sujeto es racional si la mayoría de sus creencias son racionales; una creencia es racional si está justificada; por lo tanto, 
un sujeto es racional si la mayoría de sus creencias están justificadas) y, por lo tanto, tenemos buenas razones para pensar que el internismo y el externismo epistémicos implican distintas nociones de racionalidad.

Esto último es consistente con la idea de la TDS de que hay dos maneras distintas en que los seres humanos somos racionales. Si aceptamos esto, y si aceptamos que un sujeto es racional si la mayoría de sus creencias están justificadas, entonces no parece necesario pensar que los criterios para la justificación impuestos por las posiciones epistemológicas antes mencionadas sean excluyentes. Más bien parece que cada una de ellas captura apropiadamente dos maneras en que una creencia está justificada. En otras palabras, es posible postular, en analogía con la diferencia terminológica propuesta por la TDS, una diferencia terminológica entre dos maneras de entender la noción de "creencia justificada". Así, diríamos que una creencia está

Justificada 1 sólo si es producida por un proceso generalmente confiable, i.e. eficiente para alcanzar los fines del sujeto.

$J_{u s t i f i c a d a}$ sólo si satisface los requisitos normativos para la racionalidad.

Una posible reformulación de estas ideas es la siguiente: hay dos tipos de estatus normativos asociados con el concepto de justificación, cada uno de los cuales describe (respectiva y apropiadamente) diferentes tipos de hechos normativos, los cuales están asociados con tipos de creencias diferentes que son producidas por distintos tipos de procesos de razonamiento, i.e., S1 y S2.

Para que esta tesis se sostenga es necesario mostrar, sin embargo, que, en efecto, las nociones de racionalidad $_{1}$ y racionalidad 2 y de justificación $n_{1}$ y justificación $n_{2}$ se derivan, respectivamente, del funcionamiento de S1 y S2 y que las nociones de justificación $n_{1}$ y justificación ${ }_{2}$ corresponden, respectivamente, a las nociones externista e internista de la justificación. En lo que sigue haré ver que esto es posible.

\section{La TDS, el internismo y el externismo}

Como dije anteriormente, la evidencia que arrojan los estudios en psicología cognitiva del razonamiento hace verosímil pensar que hay dos sistemas de razonamiento distintos. Como haré ver en lo que sigue, la noción de racionalidad 1 puede asociarse con el funcionamiento de S1 y con una noción externista de la justificación, y la noción de racionalidad $_{2}$ con la operación de $S 2$ y con una noción internista de la 
justificación. La hipótesis que defenderé es que cada uno de estos sistemas responde a exigencias normativas de distintos tipos, las cuales describen hechos normativos asociados con nuestras capacidades de raciocinio y con nociones distintas de racionalidad y justificación.

\section{1. El externismo y S1}

De acuerdo con la definición que proponen Evans y Over, un sujeto es racional $_{1}$ si "habla, piensa, razona, toma decisiones o actúa de modos que son generalmente confiables y eficientes para alcanzar sus fines" (1996, p. 8). Esta manera de pensar la racionalidad no exige que el sujeto ofrezca (o pueda ofrecer) un argumento en defensa de sus creencias para afirmar que es racional; es suficiente que los procesos que utiliza para razonar o tomar decisiones sean (de hecho) confiables. En este sentido, es verosímil pensar que si un sujeto es racional $_{1}$, entonces la mayoría de sus creencias están justificadas ${ }_{1}$. La pregunta es si esta última corresponde a la noción externista de justificación.

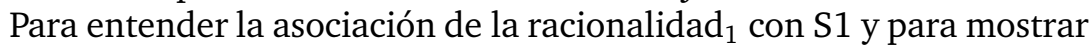
que hay una relación entre los resultados que S1 entrega y los criterios externistas de justificación epistémica es necesario subrayar algunas de las características de S1. Según la TDS, los procesos que subyacen a la operación de S1: (i) no son directamente accesibles a la conciencia; (ii) son automáticos, y (iii) son innatos, pero están modelados por nuestra interacción con el medio ambiente.

La primera de estas características se sostiene sobre la base de la evidencia que muestra que sólo los resultados finales de S1 son entregados a la conciencia. Si esto es así, tenemos buenas razones para suponer que (1) las reglas con las que opera S1 (sean éstas heurísticas, operacionales o de otro tipo) no están explícitamente representadas en el sistema y (2) aun cuando sus respuestas son sistemáticamente sensibles al contenido de los insumos que recibe, S1 no representa explícitamente estos contenidos. Esto es, tenemos buenas razones para pensar que no hay nada explícitamente representado en $\mathrm{S} 1 \mathrm{y}$, por tanto, que la información, las reglas y los procesos con los que opera S1 no son directamente accesibles a la conciencia.

Con respecto a la segunda característica mencionada, hay evidencias suficientes para pensar que las respuestas que emite S1 frente a distintos tipos de problemas de razonamiento persisten aun si el individuo trata de ignorarlos: los experimentos llevados a cabo por los psicólogos cognitivos muestran que los sujetos tendencialmente siguen ofreciendo la misma respuesta aun cuando se les haya explicado por qué ésta constituye un error. La TDS ha hecho ver, además, que en muchas ocasiones 
estos resultados sólo pueden ser corregidos o anulados si S2 los toma como insumo y los procesa. Esto, según ellos, muestra que si el razonamiento de $\mathrm{S} 1$ a veces es consistente con los principios normativos del razonamiento, ello no necesariamente se debe a que las reglas que lo caracterizan se deriven de dichos principios, sino a que hay una coincidencia (quizá azarosa) entre la respuesta pragmáticamente correcta y la normativa.

Finalmente, la idea de que S1 es innato, pero está modelado por nuestra interacción con el medio ambiente, busca explicar el hecho de que los sujetos somos sorprendentemente hábiles para resolver eficientemente los problemas que a menudo encontramos. Esto nos da buenas razones para afirmar que los procesos que subyacen a S1 toman como insumo hechos del mundo que tienen una conexión causal con su operación y es esta conexión lo que hace que ellos sean altamente confiables, i.e., tienden a proveer respuestas eficientes a problemas regularmente encontrados.

De estas características es posible concluir, por un lado, que si es cierto que los procesos que subyacen a S1 son confiables (y no necesariamente conformes con los estándares normativos clásicos de la racionalidad) y si aceptamos que un sujeto es racional 1 si (y sólo si) razona de modos generalmente confiables, i.e., eficientes para alcanzar sus fines, entonces la noción de racionalidad ${ }_{1}$ describe apropiadamente el proceder de un sujeto cuando éste utiliza su S1 para razonar. La pregunta que cabe formular ahora es si las creencias producidas por los procesos subyacentes a $\mathrm{S} 1$ pueden también ser consideradas justificadas.

Reformulemos el supuesto (b) antes mencionado para ofrecer una respuesta a esta pregunta:

$\left(b^{\prime}\right)$ un sujeto sólo puede ser considerado racional ${ }_{1}$ si la mayoría de sus creencias son racionales.

Si este supuesto es correcto, y si aceptamos que un sujeto es racional ${ }_{1}$ si la mayoría de sus creencias son el producto de procesos confiables de producción de creencias, entonces podemos afirmar que aquellas creencias que resultan de estos procesos son racionales. Sin embargo, no parece verosímil pensar que estas creencias son racionales en el sentido postulado por las teorías clásicas normativas de la racionalidad, i.e., sistemáticamente consistentes con las reglas del razonamiento correcto. Así, es razonable afirmar que son racionales porque son el resultado de procesos confiables de producción de creencias y eficientes para alcanzar nuestros fines. Una vez establecido esto y si aceptamos el 
otro supuesto antes mencionado, i.e., (a) una explicación de la creencia justificada ofrece una explicación de la creencia racional, entonces tenemos que aceptar que estas creencias son racionales sólo si están (en algún sentido) justificadas.

Acabo de decir que una creencia producida por S1 es racional porque fue producida por un proceso cognitivo confiable. Así, parece que la explicación más adecuada para la racionalidad de estas creencias es la que proviene de la noción de justificación ${ }_{1} \mathrm{y}$, por tanto, podríamos afirmar que si las creencias producidas por S1 están justificadas, ellas lo estarán por los criterios que se desprenden de esta noción de justificación. Falta hacer ver que esta noción corresponde a la noción externista.

El externismo establece que una creencia está justificada si (y sólo si) fue formada por un proceso confiable de producción de creencias. Así, parece evidente que la noción externista de la justificación se corresponde con la noción de justificación . $_{1}$. Sin embargo, esta conexión podría resultar fallida en cuanto que las nociones de confiabilidad que tienen los defensores de la TDS en mente y la que subyace al externismo epistémico no son equivalentes. Si la noción de confiabilidad que subyace a la justificación $n_{1}$ es la de la TDS, entonces no es evidente que ésta se corresponda con la noción externista.

Desde la perspectiva epistemológica, un proceso de formación de creencias es confiable solamente si tiende a producir un mayor número de creencias verdaderas que falsas y si sus insumos tienen una conexión causal apropiada con el mundo (que garantiza, en cierto grado, la operación correcta del proceso de producción de creencias). La idea subyacente a la TDS, por su parte, es que un proceso es confiable si tiene cierto valor adaptativo (i.e., si contribuye a nuestra aptitud para la sobrevivencia), lo cual no necesariamente implica que las creencias que este proceso produce sean (o tiendan) a ser verdaderas. La pregunta es si puede establecerse un vínculo entre estas dos nociones de confiabilidad tal que nos permita afirmar que la noción de justificación se corresponde con la noción externista de justificación.

La TDS afirma que la noción de racionalidad $_{1}$ se sustenta sobre el hecho de que los seres humanos razonamos de modos que no se corresponden con lo que las teorías normativas acerca de la racionalidad exigen de nosotros para afirmar que somos racionales y que, sin embargo, nos conducen a la consecución de nuestros fines. Es importante resaltar que esta afirmación captura un hecho normativo (i.e., para alcanzar nuestros fines debemos utilizar procesos confiables (en el sentido adaptativo)) que se expresa en términos de un estatus que ese hecho le confiere a nuestras creencias (i.e., una creencia producida 
por un proceso confiable está justificada ${ }_{1}$ ). Nótese que este estatus proviene de las consecuencias que tiene razonar de ciertas maneras, i.e., aquellas que son eficientes para alcanzar nuestros fines. Este mismo hecho normativo es el que captura el externismo cuando afirma que una creencia $p$ está justificada para un sujeto $S$ sólo si aceptar $p$ tiene consecuencias epistémicamente positivas para $S$. En este sentido, un primer vínculo entre las nociones de confiabilidad que subyacen al externismo epistémico y al adaptacionismo de la TDS tiene que ver con que, según ambas, las consecuencias que tiene razonar de cierto modo subyacen a algunos de nuestros juicios evaluativos respecto de las creencias o de los sujetos en general.

Ahora bien, el confiabilismo verídico afirma que las consecuencias positivas están siempre vinculadas con la obtención de creencias verdaderas (esto nos provee con una mejor garantía de que nuestros fines serán alcanzados) (Kornblith 1988). El confiabilismo adaptativo, por su parte, considera que las consecuencias positivas tienen que ver con nuestra sobrevivencia. Sin embargo, es razonable pensar que aquellos procesos que nos lleven a producir un mayor número de creencias verdaderas que falsas muy probablemente sean aquellos que (con mayor frecuencia) nos permitan ofrecer respuestas adecuadas y eficientes a los problemas que a menudo encontramos (i.e., respuestas que garanticen nuestra aptitud y contribuyan a nuestra sobrevivencia).

Así, en segundo lugar, podemos aceptar que si un proceso es confiable en el sentido epistémico, muy probablemente también lo sea en el sentido adaptativo (aunque no necesariamente tenemos que aceptar que lo mismo ocurre en sentido inverso). Si lo anterior es correcto, entonces podemos afirmar que cualquier juicio epistémico-evaluativo que se haga de los resultados que entrega S1 apela a la noción de confiabilidad epistémica, i.e., sólo aquellas creencias entregadas a la conciencia por S1 que hayan sido producidas por un proceso confiable (en el sentido epistémico) estarán justificadas ${ }_{1}$. En este sentido, el externismo nos ofrece criterios para juzgar, de entre todos los procesos que subyacen a S1, cuáles podrían ser considerados como procesos (epistémicamente) confiables.

En resumen, si aceptamos que los procesos con los que opera S1 no nos son accesibles, pero que las creencias que produce están justificadas $_{1}$, entonces tenemos que afirmar que el sujeto de la creencia no podrá acceder a las propiedades justificativas de tales creencias. Sin embargo, hay buenas razones para aceptar que razonar con S1 es racional y, por tanto, que (al menos) algunas de las creencias que produce S1 son racionales. La explicación más plausible que tenemos 
para esta noción de creencia racional es aquella que proviene de la noción externista de justificación, i.e., aquello que determina cuándo una creencia está justificada son factores que no necesariamente son accesibles al sujeto de la creencia. En otras palabras, podemos decir que la explicación más adecuada del carácter normativo de las creencias que entrega S1 como resultado es aquella que apela a los criterios de justificación provistos por el externismo epistémico. ${ }^{6}$

\subsection{S2 y el internismo epistémico}

Evans y Over afirman que un sujeto es racional $_{2}$ si "piensa, habla, razona, toma decisiones o actúa con base en ciertas razones que pueden ser sancionadas por una teoría normativa de la racionalidad" (Evans y Over 1996, p. 8). Nótese que esta manera de analizar el concepto de "racionalidad" impone exigencias al sujeto que no se desprenden de la noción de racionalidad ${ }_{1}$. La pregunta, sin embargo, es si esta noción de racionalidad tiene el mismo tipo de relación con S2 y con el internismo que la noción de racionalidad 1 con $\mathrm{S} 1 \mathrm{y}$ con los criterios externistas para la justificación.

Para ofrecer una respuesta positiva a esta pregunta es suficiente subrayar las siguientes características de S2. Este sistema, presuntamente, (a) está guiado por ciertos principios que están explícitamente representados en él; (b) entrega resultados que son sistemáticamente correctos de acuerdo con los principios normativos de razonamiento; $\mathrm{y}$

${ }^{6}$ El vínculo entre $S 1$ y la norma externista gana claridad si se piensa del siguiente modo. S1 está compuesto de muchos procesos distintos. Algunos de estos procesos son confiables (en el sentido veritista), otros no lo son. Si queremos ejercer un juicio epistémico respecto de la justificación de las creencias que S1 produce, debemos apelar a la norma externista, i.e., si $S$ está justificado (en $t$ ) en creer que $p$, entonces la creencia $p$ de $S$ debe haber sido formada por un proceso confiable, donde un proceso es confiable sólo si tiende a producir un mayor número de creencias verdaderas que falsas. Si bien el sujeto de la creencia no necesariamente está obligado a saber que está justificado en creer que $p$ (y, en este sentido, el "debe" de la norma externista no impone obligaciones a dicho sujeto en el momento de adquirir una creencia), no es el caso, tampoco, que cualquier creencia formada por

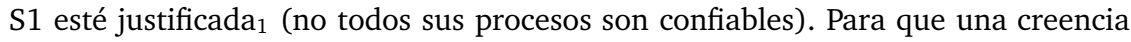
esté justificada ${ }_{1}$ es necesario que el proceso específico que la produjo satisfaga los estándares impuestos por la norma externista. Es discutible si este "debe" es meramente retórico; sin embargo, es razonable afirmar que esto no es el caso en cuanto que la norma determina cuándo una creencia puede ser aceptada, i.e., establece los criterios que un proceso debe satisfacer para afirmar que las creencias que produce están justificadas. 
(c) recibe como insumo hechos del mundo (igual que S1) y creencias producidas por los procesos subyacentes a $\mathrm{S} 1$.

La segunda de estas características nos da razones para suponer que los principios que gobiernan el razonamiento con S2 son los que las teorías normativas de la racionalidad postulan y, por lo tanto, para afirmar que si un sujeto razona con su S2, él probablemente es racional 2 . Si un sujeto es (al menos en ocasiones) racional $_{2}$, entonces (al menos) aquellas creencias que hayan sido producidas por los procesos subyacentes a su S2 estarán justificadas. Pero no podemos decir que están justificadas del mismo modo que las creencias producidas por S1, porque, como ya he dicho, estas últimas no siempre (y más bien casi nunca) satisfacen los estándares postulados por las teorías clásicas normativas de la racionalidad. Así, podemos afirmar que estas creencias están justificadas ${ }_{2}$. Falta saber si esta noción se corresponde con la noción internista.

El internismo sostiene, como dije anteriormente, que una creencia está justificada sólo si tiene una relación adecuada (desde un punto de vista epistémico) con otras creencias en un sistema y si el sujeto de la creencia puede ofrecer un argumento en su defensa (i.e., puede dar cuenta de la relación que su creencia de que $p$ tiene con sus otros estados mentales (e.g., sus otras creencias) y con el mundo). La idea subyacente a esta posición es que un sujeto sólo está justificado en creer que $p$ si tiene "buenas razones" a favor de $p$, donde "tener buenas razones" significa hacer honor a nuestra responsabilidad epistémica, i.e., reflexionar respecto de la aceptabilidad de una creencia y sólo aceptarla si hay un buen argumento que la sustente (i.e., que sea consistente con las reglas o principios de la argumentación). En otras palabras, "tener buenas razones para creer que $p$ " significa que el sujeto de la creencia puede mostrar que $p$ es consistente con el resto de sus creencias según lo establecen las reglas o principios de razonamiento (los cuales se desprenden de los principios o reglas de la argumentación). ${ }^{7}$ Así, desde esta perspectiva, un sujeto está justificado en creer que $p$ si (la mayor parte del tiempo) razona conforme lo establecen los principios de razonamiento, i.e., $S$ debe ser conciente de que su proceso de razonamiento respeta los principios en cuestión y debe poder dar cuenta de ese hecho.

${ }^{7}$ Dice BonJour (1998): "Para cualquier argumento una cuestión que es cercanamente análoga a la cuestión de la justificación epsitémica para las proposiciones puede traerse a colación: ¿hay alguna razón para pensar que la conclusión del argumento o bien debe ser verdadera o bien es probablemente verdadera si las premisas son verdaderas? [...] Cuando una razón así existe, puede decirse que el argumento en cuestión es racionalmente convincente y que la inferencia en cuestión está, en algún sentido modificado, epistémicamente justificada" (p. 5). 
Nótese que esta descripción del internismo es consistente con la caracterización que antes hice de la justificación ${ }_{2}$ (i.e., una creencia está justificada sólo si satisface los requisitos normativos para la racionalidad). Así, puede afirmarse que la explicación más adecuada del carácter normativo de las creencias que entrega S2 como resultado es aquella que apela a los criterios internistas de la justificación.

En resumen, dado que S2 es un sistema voluntario (i.e., opera cuando el sujeto considera que es necesario revisar alguna respuesta o corregir alguna otra), consciente y cuyas reglas (en la medida que están explícitamente representadas) nos son accesibles a través de la reflexión, es verosímil afirmar que muchas de las creencias que producen los procesos subyacentes a S2 estarán justificadas 2 . Además, si aceptamos que los procesos que constituyen a S2 y las reglas con que opera no sólo nos sirven como guías para decidir qué creer, sino también nos permiten satisfacer nuestras obligaciones epistémicas (i.e., aceptar creencias para las que podemos ofrecer razones), entonces podemos aceptar que la noción de justificación ${ }_{2}$ se corresponde con la noción internista de justificación.

\subsection{La "justificación", un concepto dual}

Si lo hasta aquí argumentado es correcto, entonces es razonable afirmar que las creencias formadas por S1 están justificadas por "default". Puesto que los procesos que operan en S1 son altamente confiables, no necesitamos escrutar sus resultados para estar justificados en aceptarlos. Las creencias producidas por S1 son el resultado de procesos generalmente confiables no sólo porque tienden a producir respuestas eficientes y correctas a los problemas que enfrentamos, sino también porque, a menos que estemos en una situación de duda o en la que algo muy importante esté en juego, no las ponemos en cuestión. Por otro lado, las creencias de S2 están justificadas porque son el resultado de un proceso consciente que permite revisar otras creencias (previamente sostenidas) y nos permite asegurarnos de que la creencia adquirida es consistente con ellas. Además, permite que las inferencias llevadas a cabo sean en conformidad con los principios normativos de la racionalidad.

Si esto es correcto y si aceptamos que razonar con S1 implica (la mayor parte del tiempo) ser racional $_{1}$ y que razonar con S2 posibilita ser racional $_{2}$, entonces es plausible afirmar que los resultados que entrega cada uno de estos sistemas pueden ser verosímilmente evaluados en términos de las nociones respectivas de justificación $n_{1}$ y justifi- 
cación $n_{2}$. En otras palabras, es plausible afirmar que S1 genera creencias justificadas 1 (i.e., creencias que son racionales y que están justificadas por criterios externistas); y S2 produce creencias justificadas 2 (i.e., creencias que son racionales y están justificadas por criterios internistas).

Una primera lección que puede sacarse de la TDS es que los conceptos de justificación y racionalidad son duales, i.e., las intuiciones que subyacen al internismo y al externismo capturan apropiadamente diferentes maneras de ser racional y diferentes maneras en que una creencia está justificada. Aunque esta lección es interesante, todavía falta ver si es sostenible. En particular, es necesario examinar dos supuestos que permiten que la analogía entre las nociones de racionalidad 1 y racionalidad $_{2}$, por una parte, y las de justificación y justificación $_{2}$, por la otra, se sostenga: (1) la racionalidad ${ }_{1}$ se deriva de la operación de S1 y la racionalidad $_{2}$ de $\mathrm{S} 2$; (2) las nociones análogas de justificación ${ }_{1}$ y justificación ${ }_{2}$ están asociadas a los criterios internistas y externistas para la justificación, respectivamente, y a la operación de S1 y de S2.

Evans y Over argumentan que mientras que hay una correlación fuerte entre la noción de racionalidad $_{1}$ y $\mathrm{S} 1$ y la noción de racionalidad $_{2}$ y $\mathrm{S} 2$, no es posible establecer un vínculo directo entre ellos. Esto es así puesto que: (i) "hay ocasiones en las que para ser racional $_{1}$ debes aplicar recursos conscientes o procesos del tipo 2" (Evans y Over 1996, p. 148); y (ii) también es posible "conformarse a una teoría normativa enteramente con procesos del tipo 1" (Evans y Over 1996, p. 148). Una manera plausible de entender estas afirmaciones es a través del examen de las siguientes dos tesis sostenidas por los simpatizantes de la TDS: (a) la mayoría de nuestras respuestas razonables a tareas de razonamiento (o la mayoría de los resultados inferenciales que son evaluados como razonables) no son el producto de uno solo de los sistemas de razonamiento; más bien, la mayor parte del tiempo son el resultado de la interacción entre S1 y S2. (b) No es el caso que sólo los procesos subyacentes a S1 son confiables, ni que sólo las creencias producidas por S2 son capaces de satisfacer los requisitos normativos para la racionalidad. ${ }^{8}$ En suma, no está claro que ser racional $_{1}$ necesariamente implique sólo usar $\mathrm{S} 1$, ni que ser racional $_{2}$ (necesariamente) implique sólo razonar con S2.

\footnotetext{
${ }^{8}$ Aun cuando los resultados de la operación de S1 normalmente serían mejor caracterizados por la noción de racionalidad $_{1}$, a veces también pueden ser descritos apelando a la noción de racionalidad $_{2}$ puesto que ocasionalmente satisfacen esos requisitos.
} 
Si la racionalidad ${ }_{1}$ no se deriva (exclusivamente) de la operación de $\mathrm{S} 1$ y la racionalidad ${ }_{2}$ de $\mathrm{S} 2$, entonces el primer supuesto que permite que la analogía se sostenga parece ser falso. Sin embargo, para los propósitos de este trabajo (i.e., para afirmar que la noción de justificación es dual y que, por tanto, no hay una oposición genuina entre el internismo y el externismo epistémicos) es suficiente que el segundo supuesto se sostenga, i.e., es suficiente que S1 sólo entregue como resultado creencias justificadas $_{1}$ y que S2 pueda entregar creencias justificadas 2 . En lo que sigue haré ver que sólo S2 puede conferir justificación ${ }_{2}$ a nuestras creencias y, en consecuencia, que este supuesto se sostiene.

\subsection{S1, S2 y la justificación epistémica}

Como argumenté anteriormente, la noción de justificación $n_{2}$ es internista, i.e., la creencia $p$ de $S$ puede considerarse justificada 2 sólo si $S$ reconoce, a través de la reflexión (i) la verdad del hecho de que su creencia de que $p$ es probablemente verdadera dada su evidencia total; o (ii) que su creencia de que $p$ sea consistente con sus otras creencias (previamente sostenidas). La idea es que $S$ no sólo debe tener acceso a las propiedades que confieren justificación a la creencia, sino también a las reglas que establecen la permisibilidad de dicha relación inferencial $y$, con base en ellas, debe evaluar el resultado de su proceso de razonamiento. En otras palabras, una creencia puede considerarse justificada si, al aceptarla, $S$ satisface sus obligaciones epistémicas o su responsabilidad como una criatura racional y, para que esto último sea el caso, es necesario que $S$ tome una posición reflexiva respecto de las razones que sostienen a una creencia justificada.

Desde la perspectiva de la TDS, una de las funciones de S2 es hacer explícitas las representaciones de S1, haciéndolas así accesibles a la conciencia a través de la reflexión. La idea de fondo en este planteamiento es que los sujetos pueden acceder a los contenidos sobre los que opera S2 y a las reglas que lo gobiernan, puesto que ambos están explícitamente representados en él. Si esto es correcto y si aceptamos que S2 puede recibir como insumo hechos del mundo $y$ creencias que provienen de S1, entonces podemos aceptar que S2 nos permite reflexionar sobre los resultados que S1 entrega a la conciencia y, en este sentido, nos permite preguntarnos respecto de la consistencia que dichos resultados tienen (o pueden tener) con los principios normativos de razonamiento postulados por las teorías normativas de la racionalidad. En otras palabras, las razones que tenemos para aceptar las creencias producidas (o procesadas) por S2 nos son accesibles de un modo que 
no es el caso para el contenido de S1. Si esto es correcto y si aceptamos que los resultados emitidos por S2 son sistemáticamente consistentes con los estándares normativos (idealizados) de racionalidad, entonces podemos aceptar que las creencias producidas por dicho sistema (las creencias justificadas $_{2}$ ) satisfacen los criterios internistas para la justificación, i.e., tienen un estatus normativo proveniente de sus relaciones con otras creencias o estados mentales que pueden ser o bien un producto de S1 o de S2 mismo.

Ahora bien, S2, a diferencia de $\mathrm{S} 1$, es un sistema consciente, i.e., los sujetos pueden acceder a los contenidos con los que opera y a las reglas que lo gobiernan. Así, sólo S2 puede revisar si una nueva creencia (producida por S1 o por S2 mismo) es consistente con el resto de nuestras creencias. Además, en caso de que un sujeto se percate de que está utilizando una regla incorrecta, sólo S2 puede detener el proceso de razonamiento y/o rechazar la creencia en cuestión. En otras palabras, sólo si un sujeto utiliza su S2 es capaz de cumplir con su obligación epistémica y, en este sentido, podemos aceptar que las reglas que constituyen a S2 sirven como guía para el buen razonamiento. Si esto es el caso y si aceptamos que una creencia está deontológicamente justificada sólo si el sujeto de la creencia es conciente de su deber (u obligación) epistémico y cumple con él, entonces tenemos que aceptar que sólo aquellas creencias que fueron producidas o procesadas por S2 pueden satisfacer los requisitos de la justificación propuestos por el internismo.

S1, por su parte, no es un sistema reflexivo. Como ya dije, no hay nada explícitamente representado en S1 y, por lo tanto, no hay nada sobre lo cual reflexionar cuando razonamos con él. S1 no es capaz de evaluar sus operaciones ni sus resultados: no accede a las reglas con que opera ni a los contenidos de las creencias que procesa y, por tanto, no es capaz, por sí solo, de revisar la consistencia de las creencias que entrega como resultado con el resto de las creencias del sujeto. Además, sólo ocasionalmente entrega resultados que están de acuerdo con los requisitos normativos impuestos por las reglas del razonamiento correcto. Resulta muy inverosímil afirmar que un sistema de cuya operación no somos conscientes y al contenido del cual no tenemos acceso pueda ser apropiadamente descrito como un sistema reflexivo, i.e., un sistema capaz de conferir justificación . $_{\text {. }}$

En resumen, si la reflexión es una condición necesaria para la justificación $_{2}$, entonces no es posible afirmar que S1 puede conferir justificación $_{2}$ a las creencias que produce. Las creencias de S1 sólo podrían considerarse justificadas $_{2}$ si el sujeto pone a funcionar su S2 para que éste 
las procese, pero entonces diríamos que estas creencias son el resultado de S2 o de la interacción de S1 y S2 (i.e., no de S1). Si esto es así, entonces el segundo supuesto (i.e., $\mathrm{S} 1$ sólo puede conferir justificación a las creencias que produce y la justificación ${ }_{2}$ proviene exclusivamente de la operación de S2) se sostiene y, por tanto, es plausible afirmar que las intuiciones que subyacen a las posiciones internistas y a las externistas son necesarias para entender apropiadamente el fenómeno de la justificación epistémica.

\section{Conclusión}

Las exigencias que imponen los conceptos deontológico y consecuencialista de "justificación" son de distintos tipos, pero esto no implica que haya una oposición real entre ellas. La pregunta con respecto a qué estatus normativo le confiere la "justificación" a nuestras creencias no es una pregunta disyuntiva. Si tomamos en cuenta la manera como de hecho funciona la mente humana y si consideramos que la TDS es una teoría verosímil, entonces el problema de la justificación epistémica puede reformularse en términos del establecimiento de criterios complementarios que ayuden a explicar y sustanciar la noción de "creencia justificada" con base en los procesos que de hecho utilizamos y las reglas que pueden servir como guía para ser mejores agentes cognitivos.

En otras palabras, si lo que aquí he argumentado es correcto, entonces el problema de la justificación epistémica no necesariamente debe formularse en términos de una elección de dos explicaciones alternativas: o bien nuestras creencias son el producto de (i) procesos particulares que caracterizan un mecanismo de razonamiento (S1) que no puede satisfacer (sistemáticamente) los requisitos impuestos por una noción internista de justificación; o (ii) de ciertos otros procesos que caracterizan a un mecanismo de razonamiento (S2) cuyo fin central es ofrecer respuestas en conformidad con los requisitos normativos clásicos (con la consecuencia de que tales creencias son racionales en virtud de sus relaciones internas con otras creencias o estados mentales). Más bien, la justificación epistémica tiene fuentes distintas y el establecimiento de criterios para atribuirla debe tomar en cuenta este hecho si pretende capturar adecuadamente el fenómeno mismo de la justificación. 


\section{BIBLIOGRAFÍA}

BonJour, L., 2005, "Internalism and Externalism", en P.K. Moser (comp.), The Oxford Handbook of Epistemology, Oxford University Press, Oxford, pp. 234263.

- 1998, In Defense of Pure Reason, Cambridge University Press, Cambridge. Cohen, J., 1986, The Dialogue of Reason, Clarendon Press, Oxford.

Eraña, A., 2007, "Sobre la viabilidad de una epistemología empírica y normativa", Signos Filosóficos, vol. 9, no. 17, pp. 101-138.

Evans, J., 2006, "Dual System Theories of Cognition: Some Issues", Proceedings of the 28th Annual Meeting of the Cognitive Science Society, Lawrence Erlbaum, Vancouver, pp. 202-207.

_, 2003 , "In Two Minds: Dual-Process accounts of Reasoning", Trends in Cognitive Sciences, vol. 7, no. 10, pp. 454-459.

_ 1984, "Heuristic and Analytic Processes in Reasoning", British Journal of Psychology, vol. 75, pp. 451-468.

Evans, J. y D. Over, 1996, Rationality and Reasoning, Erlbaum Psychology Press, Hove, Reino Unido.

Foley, R., 2002, "Conceptual Diversity in Epistemology", en P.K. Moser (comp.), The Oxford Handbook of Epistemology, Oxford University Press, Oxford, pp. 177-203.

Goldman, A.I., 1979, "Reliabilism. What is Justified Belief?", en G.S. Pappas (comp.), Justification and Knowledge, D. Reidel, Dordrecht.

Griggs, R., 1983, "The Role of Problem Content in the Wason Selection Task and THOG Problem", en J. Evans (comp.), Thinking and Reasoning: Psychological Approaches, Routledge and Kegan Paul, Londres, pp. 16-43.

Kahneman, D., P. Slovic y A. Tversky (comps.), 1982, Judgment and Uncertainty: Heuristics and Biases, Cambridge University Press, Cambridge, Mass.

King, P., 2000, "Internalismo, externalismo y autoconocimiento", Crítica. Revista Hispanoamericana de Filosofía, vol. 32, no. 96, pp. 99-119.

Kornblith, H., 2001, "Internalism and Externalism: A Brief Historical Introduction", en H. Kornblith (comp.), Epistemology: Internalism and Externalism, Blackwell, Oxford, pp. 1-9.

_, 1988 , "What Is Naturalistic Epistemology?", en H. Kornblith (comp.), Naturalizing Epistemology, The MIT Press, Cambridge, Mass., pp. 1-14.

Lopes, L., 1991, "The Rhetoric of Irrationality", Theory and Psychology, vol. 1, no. 1 , pp. 65-82.

Manktelow, K. y J. Evans, 1979, "Facilitation of Reasoning by Realism: Effect or Non-Effect", British Journal of Psychology, vol. 70, pp. 477-488.

Moser, P.K., 1956, Empirical Justification, D. Reidel, Dordrecht.

Plantinga, A., 1993, Warrant: The Current Debate, Oxford University Press, Oxford.

Sloman, S.A., 1996, "The Empirical Case for Two Systems of Reasoning", Psychological Bulletin, vol. 119, no. 1, pp. 3-22. 
Sosa, E., 2002, "Dos falsas dicotomías: fundamentismo/coherentismo e internismo/externismo", en J. Beltrán y C. Pereda (comps.), La certeza ¿un mito? Naturalismo, falibilismo y escepticismo, Instituto de Investigaciones Filosóficas-UNAM, México.

Stanovich, K.E. y R.F. West, 2000, "Individual Differences in Reasoning: Implications for the Rationality Debate?", Behavioral and Brain Sciences, vol. 23, no. 5, pp. 645-726.

Stein, E., 1996, Without Good Reason, Clarendon Press, Oxford.

Tversky, A. y D. Kahneman, 1983, "Extensional versus Intuitive Reasoning: The Conjunction Fallacy in Probability Judgment", Psychology Review, vol. 90, no. 4, pp. 293-315.

_ 1982 , "Judgment under Uncertainty: Heuristics and Biases", en Kahneman, Slovic y Tversky 1982, pp. 3-22.

Wason, P.C., 1969, "Regression in Reasoning?", British Journal of Psychology, vol. 60, pp. 471-480.

_, 1966, "Reasoning", en B.M. Foss (comp.), New Horizons in Psychology, Penguin, Harmondsworth, pp. 135-151.

Wason, P.C. y P. Johnson-Laird, 1972, Psychology of Reasoning, Harvard University Press, Cambridge, Mass.

_ 1970, "A Conflict Between Selecting and Evaluating Information in an Inferential Task", British Journal of Psychology, vol. 23, pp. 63-71.

Williams M., 2001, Problems of Knowledge. A Critical Introduction to Epistemology, Oxford University Press, Oxford.

Recibido el 25 de febrero de 2008; aceptado el 4 de agosto de 2008. 